International Journal of Pharmaceutics \& Pharmacology

\title{
Post-Market Safety and Efficacy Surveillance of Herbal Medicinal Products from Users' Perspective: A Qualitative Semi-Structured Interview Study in Kumasi, Ghana
}

\author{
Adusei-Mensah Frank ${ }^{1}$, Haaranen Ari ${ }^{2}$, Kauhanen Jussi ${ }^{1}$, Tikkanen-Kaukanen Carina ${ }^{3 *}$, Henneh Isaac \\ Tabiri $^{4}$, Ganu Daniel ${ }^{5}$, Ametsetor Ebenezer Seklu Edem ${ }^{6}$, Olaleye Sunday Adewale ${ }^{7}$ and Ekor Martins ${ }^{4}$
}

${ }^{1}$ Institute of Public Health and Clinical Nutrition, School of Medicine, University of Eastern Finland, Finland

${ }^{2}$ Department of Nursing Science, University of Eastern Finland, Kuopio, Finland

${ }^{3}$ Helsinki Institute of Sustainability Science and Ruralia Institute, University of Helsinki, Finland

${ }^{4}$ Department of Pharmacology, School of Medical Sciences, University of Cape Coast, Cape Coast, Ghana

${ }^{5}$ School of Postgraduate Program, Adventist University of Africa, Nairobi, Kenya

${ }^{6}$ Department of Biochemistry, School of Biological Sciences, University of Cape Coast, Cape Coast, Ghana

${ }^{7}$ Department of Marketing, Management and International Business, University of Oulu, Finland

Article info

Received 01 July 2019

Revised 26 July 2019

Published 31 July 2019

*Corresponding author: Carina Tikkanen-Kaukanen, Helsinki Institute of Sustainability Science and Ruralia Institute, University of Helsinki, Finland; E-mail: tikkanen-kaukanen@helsinki.fi

\begin{abstract}
Objective: Despite the increased patronage of herbal medicinal products (HMP), they remain poorly regulated in Ghana and their efficacy and safety data is seldom adequate. The objective of the present study is to provide insight into concerns regarding post-market HMP safety and efficacy from the perspectives of users in Kumasi, Ghana.

Methods: Thirty-seven randomly selected HMP consumers in Kumasi, were interviewed using semi-structured questionnaire. Non-users of HMPs and users less than 18 years were excluded from the study. Mixed method sampling method was used for the study.

Results: HMPs were reported to be gentle and safer than orthodox medicines by informants. Only 19\% of informants voluntarily reported HMP use to their general practitioner. Informants (94.6\%) experienced minimal effects, whiles (5.4\%) experienced moderately severe adverse effects.

Conclusion: It was observed from the study that some HMPs are however non-effective and harmful. We would suggest that HMP users should exercise caution with regards to where to buy and when using HM. "Shyness" and the "feeling of being condemned by the physician" were identified to be the possible causes of the communication barrier between the physicians and the HM users. We recommend physicians to initiate discussion on this topic during consultations and guide users on safe use of these HMPs.

Keywords: Experience; Participants; Communication; Interview; Herbal medicine; Herbal medicinal product
\end{abstract}

\section{Abbreviations}

ACT: Artemisinin Based Combination Therapy; FDA: Food and Drug Administration; GH: Ghana New Cedi; GP: General Practitioner; HMPs: Herbal Medicinal Products; HMs: Herbal Medicines; NHIS: National Health Insurance Scheme; RQDA: R Package for
Computer Assisted Qualitative Data Analysis; SPSS: Statistical Package for Social Sciences; TAMPC: Traditional and Alternative Medical Practice Council; UCCIRB: University of Cape Coast Institutional Review Board; USD: United States Doller; WHO: World Health Organization. 


\section{Introduction}

The use of herbal medicines (HMs) is widespread. Herbal medicinal products (HMPs) are usually used for preventive, curative and for chronic disease management $[1,2]$. The surge in patronage in the last decades is thought by some researchers to be due to the perceived efficacy and increased trust in the HMs $[3,4]$. Despite the perceived efficacy, available scientific evidence questions the efficacy of HMs. Bent [5] found little scientific evidence regarding the efficacy and safety of most of the over 2000 HMPs available and consumed in the US. Recently, Wilmot and colleagues discovered that all the top-5 most patronized antimalarial herbal preparations of the Greater Accra, Western and Central regions of Ghana had efficacy levels below the WHO standard [6]. In the same study, $40 \%$ of the top- 5 antimalarial preparations were found to have been adulterated with chloroquine.

There is a huge public misconception regarding the safety of HMs as many construe 'natural' as 'safety' $[7,8]$. There are problems with batch variations, dosage and concentration inconsistencies of the active ingredients with some HMPs on the market [9]. A case series study in Belgium on Chinese herbal product for weight loss revealed that the product led to the development of end-stage renal failure, urothelial carcinoma and prophylactic kidney removal [10]. In another study, $28.8 \%$ of herbal preparations investigated were found to be contaminated with heavy metals and $7 \%$ of the herbal products were consciously adulterated with conventional drugs to improve efficacy [11].

HMs though, are perceived to be generally safe, they can pose health risk to consumers [12]. There have been reports of deaths and adverse effects such as cardiotoxicity, psychosis, hepatotoxicity and nephrotoxicity after administration of HMs [13-15]. HM and its supplements account for 25\% of druginduced acute liver damage that result in liver transplant or death in the US [16-18]. Inherently toxicity, deliberate or inadvertent adulteration, microbial contaminants and pesticides have been linked with toxicities reported with HM usage [17]. Current policies safeguard not the safety of patients from potentially unsafe HMPs [18]. In Ghana, effort is being made by regulatory authorities to register HMPs before they are introduced into the market to safeguard public health. As part of the effort, the Traditional and Alternative Medical Practice Council (TAMPC) was established with the mission of promoting, controlling and regulating the practice of Traditional and Alternative
Medicine in Ghana. Despite the creation of TAMPC, many HMPs still get to the market through the backdoor and without safety evaluation. There has not also been regular field post-market safety and efficacy surveillance on both registered and unregistered HMPs in the market thereby creating a major public health concern.

In Ghana, most consumers fail to report cases of adverse health effects associated with the use of HMPs and herb-drug interactions there is also lack of zeal and motivation on the part of the consumer or lack of formal systems established by authorities to encourage the reporting of such cases. There is, therefore, paucity of data on HMPs associated adverse health reactions and consequences of their interaction with food and orthodox medicines in Ghana. The true state of side effects for most HMPs on the Ghanaian market is unknown, requiring the need for regular monitoring studies [5]. The primary aim of this post-market surveillance, therefore, was to obtain in-depth understanding and insight into concerns regarding efficacy and safety of HMPs from the perspectives of the consumers.

\section{Methods}

Pre-study feasibility interviews were carried out on 10 participants (informants) prior to the main study to finetune the interview guide. Transcripts of the pre-study trials were not added to the main study. In the main study, face-to-face semi-structured interviews were conducted on 37 information-rich users of HMPs (age $\geq$ 18 years). Interviews were conducted with the help of an interview guideline.

\section{Sampling method}

A simple random and cross-sectional methods were used for this study. Herbal centers and clinics were randomly selected. Herbal medicine users were randomly recruited from the streets and central market of Kumasi. At the herbal clinics, all patients who patronized the centers between 10:00 to 16:00 from Monday to Friday during the study period (4th July to $12^{\text {th }}$ August 2017) were contacted and those who met the inclusion criteria and agreed to partake in the study were recruited (opportunistic/cross-sectional method). To be recruited in the study, the individual needed to be 18 years or over, a user of HMPs, agree and sign a consent form and being able to communicate in at least English or Akan language. Background to the study was provided in the invitation letter including the need to 
grant 30 minutes interview time if they agreed to participate in the study. The HMPs users who agreed to participate in the study upon receipt of the invitation letter were given consent form. The form provides detailed information about the study, voluntary participation and freedom to opt out at any time of the study. Possible risks and benefits of participating in the study were also detailed in the form. The interviews were conducted in English or Akan language depending on the preferences of the interviewees. Interview questions were structured in order of topic and were predominantly open-ended questions. The face-to-face interview lasted for about 25-30 minutes. The interviews were supplemented with audio recordings which were later transcribed into text for analysis. Ethical clearance for study was issued by the University of Cape Coast Institutional Review Board (UCCIRB) (UCCIRB/EXT/2017/07).

\section{Data analysis}

The closed-ended questions including sociodemographic data in the study transcript were analyzed with IBM's SPSS version 21 using frequency descriptive statistical analyses. The coding and code classification feature of RQDA ( $R$ package for computer-assisted qualitative data analysis) version 3.4.4 run on R-studio 1.0.153 platform and the networking feature of Gephi were explored for the thematic qualitative data analysis. Braun and Clarke's six steps to thematic analysis process were followed $[21,22]$. The interview transcripts were organized into two levels of organization; codes and code categories (themes). Transcribed data were read severally by the researcher for familiarization with the data. A total of 47 codes with 365 initial markings were made after the first coding stage (Table 1).

Table 1: Thematic analysis process followed.

\begin{tabular}{|l|l|}
\hline \multicolumn{1}{|c|}{$\begin{array}{c}\text { Thematic analysis } \\
\text { Process [19] }\end{array}$} & \multicolumn{1}{c|}{ Activity performed } \\
\hline Familiarizing with data & Transcribing data, reading and rereading the data, noting down initial ideas. \\
\hline Generating initial codes & Reading and re-reading led to the generation of a total of 365 codes \\
\hline Searching for themes & The generated codes were grouped under initial 47 code categories (themes). \\
\hline $\begin{array}{l}\text { Defining and naming } \\
\text { themes }\end{array}$ & $\begin{array}{l}\text { A total of } 10 \text { code categories (themes) were finally generated after the continual } \\
\text { analysis, defining and refining the specifics of each theme and the overall story and the } \\
\text { aim of the study. The final codes were also stratified based on income level and sex of } \\
\text { respondents }\end{array}$ \\
\hline Producing the report & The final findings were compiled into writing the report. \\
\hline
\end{tabular}

Mixed inductive and deductive approaches to thematic analysis were used whiles following the six-step process by Braun and Clarke [19]. Most themes were inductively generated entirely from participants' responses whiles some few priori deductive themes from previous research were added. The themes (code categories) and codes were revised to contribute in answering the research question; some themes (code categories) and codes were deleted, merged and or renamed to best suit the study (Table 1 and 2). Recoding, renaming, dropping or adding of codes and code categories continued during the preparation and drafting of the results. Case categorization was done on the transcripts to categorize the transcripts based on sex and income level for easy case retrieval of the opinion of male or females and the effect of income on a theme. Sox major themes with sub-themes emerged from the study (Figure 1). Interviewees were randomly given codes ranging from $\mathrm{H} 1$ to $\mathrm{H} 37$ for anonymity. From hence, interviewees are represented in the text with these codes H1, H2....H37.

\section{Results}

\section{Socio-demographic characteristics of participants}

Most participants (59.5\%) were males, informants aged from 23 to 80 (mean age $47.8 \pm 14.2$ ). Most participants were educated; $18.9 \%$ had tertiary education, $40.5 \%$ had received either secondary, A-level, O-level or equivalent form of education, $27 \%$ had received primary level of education while the rest had no formal education. In this study, $27 \%$ of the participants lived below the international poverty line of GHC 342 per month ( $\$ 1.9$ per adult a day of the World Bank's international poverty line at the time of the study using Bank of Ghana rate). The remaining $73 \%$ had monthly incomes either equivalent to or above the international poverty line. Over $81 \%$ of informants reported to have never drank alcohol before, $16 \%$ drink moderately 
during occasions or before meals while only $3 \%$ reported of going binge sometimes. None of the 37 interviewees reported having smoked before. Over $70 \%$ of the informants have used HMPs for 2 or more years, and $73 \%$ of these individuals use HMPs routinely with or without conventional medicine for their health care.

\section{Theme 1: Efficacy of herbal medicine-(efficacy)}

The expression and rating of efficacy in real life situations were difficult with some elements of subjectivity and relativity especially for medicines with holistic effects. Four sub-themes evolved from efficacy.

\section{Efficacy from user s' perspective-(Experienced)}

Many informants $59.46 \%$ believed the HMPs they used were efficacious. "When I was a kid, an herbal product was specifically prepared for me for skin problem and it really worked for me. The skin problem has never return" (H6). Others opined that, though HMPs are effective, the efficacy is only realized if you buy a good HMP. "HMP is effective, should you get a better one, but if not, it will give you problems and will not work" (H13).

Eleven $(29.7 \%)$ users believed HM is more effective than orthodox medicine. "I used it to heal my mum when she was sick and was not responding to allopathic treatment and pharmacy drugs" (H37). "Herbs work better than pharmaceuticals" (H19). Slow but sure: Some users believed HM has long-lived effect and cure their illness, but it takes a longer time to realize the effect compared to orthodox medicine while orthodox medicine has a short-lived effect. "HMP takes long time to see the effect but it can cure the sickness completely, pharmacy drug is quick, but the sickness might come back" (H33). "My mother was cured by herbal medicine after several hospital attempts" (H5). 'Like my pressure, if I take the doctors' medicine it works but to return shortly after I stop using the drug. On the contrary, it takes about 6 months for it to return after using herbal medicine, HM really works; herbs can cure but pharmacy drug is for management"' (H36). " "...the doctor's medicine works but the sickness comes back shortly thereafter' (H28).

\section{Signs of HM efficacy; users' experience-(Signs)}

Informants in this study used different endpoints/ signs to express HMP efficacy. "My blood sugar level reduced a little after taking the HMP (observed through regular diabetes check-up in a public hospital)" (H3). "My ulcer stopped in two months instead of the threemonth timeline for treatment" (H24). Interviewees H18 and $\mathrm{H} 20$ also reported that, 'whenever they had malaria and they administered HM, it stopped'. Others used unusual signs and belief to express HM efficacy. "I started sweating after taking it and I believed it is a sign that it is working"' (H26). "The pain increased after HM administration and I believed it to be a sign of healing the pain; for a HM to heal, it will first increase the problem and then heal them all" (H16).

\section{Negative perspective on efficacy of herbal medicine- (Ineffective)}

Six users (16.2\%) were dissatisfied with the HMP they used. "HM have not helped me, I have used many, but less help, they are not effective, most of the distributors' claims are not true"' (H35). "HM has not helped me at all, it did not work for me at all!" (H27). "They brag a lot on the radio and charges a lot, but their herbs do not work for me, ...they are too expensive, and they do not work either' (H29).

\section{Theme 2: Assessing cost and cost-effectiveness of HMPs-(Cost-effectiveness)}

Two sub-themes emerged under how users see the cost of herbal products they used, namely cost of herbal medicine and cost-effectiveness from users' perspective.

\section{Cost of herbal medicines-(Cost)}

There were two different opinions on cost; the first opinion was that HMs are generally less expensive and $21.17 \%$ users have such opinion. "The HMs are usually within my means, I think they are even a bit cheap" (H9). "It is always given to me by a friend, I don't buy them, I just give him something small (small money) for transportation" (H4). "HMs are less expensive", said H17. The second group of the interviewees saw HMs to be slightly expensive. "Some HMPs are a bit expensive", (H20). "...they are expensive, some are around $\mathbb{C} 200 \mathrm{GH}$ " (about $50 \$$ USD) (H25). "Some are very expensive, too expensive, they have made me poorer"' (H29).

\section{Cost-effectiveness from user's perspective-(Cost- effective)}

Considering the per-unit cost and the realized efficacy of HMs by users, some users believed HMs are less cost-effective. "Yes, I experienced mild stroke, went to the hospital about 4-times with two different laboratory tests, but there was no good outcome. But after switching to HM, I can now write and do most of the things I used to do again and are less expensive too" (H22). "I mostly buy moderate ones, HMPs are very 
effective. I use it for all kinds of my health problems. Even yesterday I was feeling uneasy but after taking Adwutwumwaa capsules, I am now okay', (H30). A few informants believed that, the HMPs they used were not cost-effective. "It did not help me in anyway; they have also ended up taking all my pension money as a retired teacher without the sickness getting better" (H27). "No, it did not help me, used many but not much help. There had been a time I walked out of an herbal clinic when I was having an eye problem and they said I should pay $\mathbb{C} 700 \mathrm{GH}$ (175 USD) before they start the medication"' (H35).

\section{Theme 3: Users' belief and perception about herbal and orthodox medicines-(Belief and perception)}

Many interviewees believed HMs are more effective than orthodox medicines and that HMs can cure the sickness while orthodox medications are for management. They also iterated that HMs are a "slow but sure" way of treatment while orthodox medicines are quick in action but have short-lived effects. Comparison of the efficacy of the two has been detailed above under efficacy theme. Some users also opined that, HMs are natural and safer than pharmacy drugs.

HMPs are natural, non-chemical and safer than pharmaceutical medicine-(Non-chemical)

Thirty-five percent believed HMPs are natural, nonchemical and safer than orthodox medicines. "It (HMPs) is naturally extracted from plants and we believe it is more effective with less bad side effects" (H11). "I believe the components are natural, so safe" (H12). "It is good for human consumption, because it helps the human system" (H8).

\section{Trust in HMs and pharmaceutical medicine (Trust)}

Trust in HMs has increased over the years based on many reasons and a few were shared by interviewees. HMs are safer and efficacious than orthodox medicine have been discussed previously and were also seen as factors for increased trust in HMs. In addition, dissatisfied with conventional medicine and tradition handed down were identified as other contributing factors. Trust in HMs come at the expense of decreased trust in conventional health care system. Eleven informants $(29.73 \%)$ had such perception. "For some sicknesses, the doctor's medicines do not work but some herbal medicines do" (H36). "If I use HMPs, it helps me a lot, so I use it based on previous experiences because the doctors' medicine works but the sickness comes back shortly thereafter" (H28). "Most of the pharmaceutical medicines do not give you the results you are looking for, that made me go for herbal medicine"' (H22).

\section{Herbal medicine is a tradition handed down- (Tradition)}

A class of users (27.03\%) had built trust in the HMs and feel at home using HMs, believing that it is a tradition handed down from their parents and grandparents. " $\mathrm{HM}$ is the tradition of our grandparents, so we just feel at home when using it" (H2). "Cultural linkage from our parents and grandparents, when we were kids, our grandparents were giving us 'dudo' (a concoction or stock of herbal preparation which is home prepared, reheated every morning and used regularly by all members of the family)" (H1). "... from an herbalist grandfather, I use it because we believe they do not have bad effects like the pharmacy drugs." (H15).

\section{Theme 4: Concerns about and fake sub-standard HMPs-(Sub-standard)}

Fake or sham preparation is a major concern for both herbal and orthodox medicines especially in developing countries. Fake and adulteration of HMPs was seen by many interviewees to be a major concern and some informants shared their displeasure.

\section{Fake and adulterated HMPs-(Fake)}

"There are also fake HMPs on the market that is why the Ghana Food and Drugs Authority (FDA) should do a serious test... The FDA should help clean the market of fake drugs" (H1). "My concern is that there are fake ones, if you get the right HMP, it works" (H19). "I suspected an herbal mixture I took to have been adulterated, the taste and smell felt like it has been. It tasted like there were paracetamol and 'biiko' (the common name in Ghana for B-complex vitamin tablet) inside" (H24).

\section{Proliferation of HMPs primarily for business and profit-(Proliferation)}

Some informants believed that, some HMP producers are into the herbal industry solely for monetary gains and less concerned about the health of the patients. "Many just want to make money; they pick any herb and call it HM" (H36). "There is no sanity these days in the market, everyone is selling or making HMP, they are much concerned about making money and not about healing people" (H23). "My concern is that there are too many HMPs on the market, they have been in-fluxed with fake ones"' (H1).

Concerns about claims by HM vendors-(Claims) 
It was observed that some informants had misgivings concerning the claims of the HMPs' vendors. "The vendors brag a lot on the radio and charge a lot of money, but their herbs do not work for me, they sometimes lie about free screening, but you may leave there paying about $\mathbb{C} 500$ GH (\$125 USD)!'” (H29). "There are a lot of fake HMPs, not all are true, not effective, most of their claims are not true", (H35). Interviewees $\mathrm{H} 10$ and $\mathrm{H} 32$ complained that some vendors sometimes give false information to the public.

\section{Theme 5: Safety concerns of HMPs-(Safety concerns)}

Five sub-themes emerged as we discuss the safety of herbal medicines.

\section{The practice of combined herb-drug administration by users-(Poly-pharma)}

According to some users, HMs cure diseases but at a slow pace, so they administer HMPs after orthodox medical treatment to completely cure their sickness. "I do not usually combine different medications, but if I do, I first ask or request from a doctor or vendor before doing it (H7). "I use HMP and ACT combination therapy for malaria"' (H12).

\section{Adverse effects of HMs from users' experience- (Adverse events)}

Adverse health effects encountered by some informants were reported by $27.03 \%$ of interviewees. "I have now developed swollen stomach and have kidney problems according to my doctors report which hitherto were not there" (H25). "I experienced an increase in a heartbeat when I ate shortly after herbal medicine administration" (H9). "It has increased my blood pressure, ... it made my sickness go up, it could have killed me!''(H27). Other reported side effects were headache and dizziness (H10 \& H35), stomach pain and nausea (H11), migraine (H29) and constipation (H34).

\section{Adverse health effects of HMs based on hearsay- (Hearsay)}

There are several unconfirmed 'hearsays' about HMs in Ghana, and below are few of such reports from informants. "I know of a man who has been in the UK for many years, but when he came home (Ghana) for a short stay, a HMP was given to him for piles which he took orally. Shortly then after he complained of excruciating pains in the mouth, the tongue started to swell and was coming out. He died shortly after being admitted into a hospital" (H1). "You sometimes hear reports of adverse health reactions emanating from
HMPs usage especially from those bought in buses" (H13).

\section{Labelling and dosage concerns of HMPs-(Labelling)}

Labelling and dosage information are crucial for safe use of medications. Some users had concerns with labelling of some HMPs on the market. "Sometimes the labels give ambiguous statements; not clear statements. Also, they claim one HMP can heal many diseases, it is my concern', (H12).

Some important dosage lapses evolved from the reports of $24.32 \%$ informants; " "you can drink (HMs) as much as you want without any side effects" (H14). "The spirits direct me to the dosage to use, the spirits direct me to the herbal plant (medicine) to administer even to others, they direct the dosage to give and they never make mistake"'(H37).

\section{Other safety concerns raised-(Emerged)}

Other safety issues emerged during the interviews from the reports of 8 interviewees. "My only concern is the pesticides they use these days in spraying the plants, some farmers just spray any amount on the field without much knowledge like me. This issue raises concern about HMPs' safety these days. The pesticides could be blamed for all these sicknesses"'(H2). "Some of the herbal medicine may be harmful somehow because they stay too long in the shells at the shops"' (H14).

"You may die from pharmaceutical medicines overdose ... but you will never die from HMs overdose. Michael Jackson is an example of pharmaceutical drug overdose" (H9).

\section{Theme 6: Reporting usage and safety issues to General Practitioner-(Reporting to GP)}

About half of users said they will report adverse effects and usage of HMPs to the general practitioner (GP) while only $19 \%$ said they will not report adverse effects to the GP. On the contrary, most informants said they will not voluntarily tell the GP that, they are using HMs; they either felt shy or afraid of being condemned by the GP. Three sub-themes evolved under this theme.

\section{Reporting HMP usage to the GP-(Feeling shy)}

Free flow of information is crucial for patients to be guided about the safe and rational use of HMPs. Doctors, according to interviewees, usually failed to ask them if they were using HMs during hospital visits, although the HMs users (10/37) were willing to disclose such information when asked. "If the GP asks, I will tell him, though I feel shy to freely tell him myself and 
afraid of being condemned", (H27). "If the GP asks me, I will tell him but if he doesn't ask me, I will not', (H14). "I will so that the GP can advise me whether to continue or discontinue using the HMP"' (H36).

\section{Condemned by general practitioners for using HMs- (Condemning)}

Some users felt that the GP will condemn them and the HMP they are using if they should tell the GP during hospital visitation. "Sometimes you tell the doctor and he reply by saying, HMPs are not good. As for the doctors, they will condemn you and the HMP you are using" (H1). "I feel shy. I feel that the doctor will condemn me for using HMP"' (H27). "I feel the doctors will insult me", (H29).
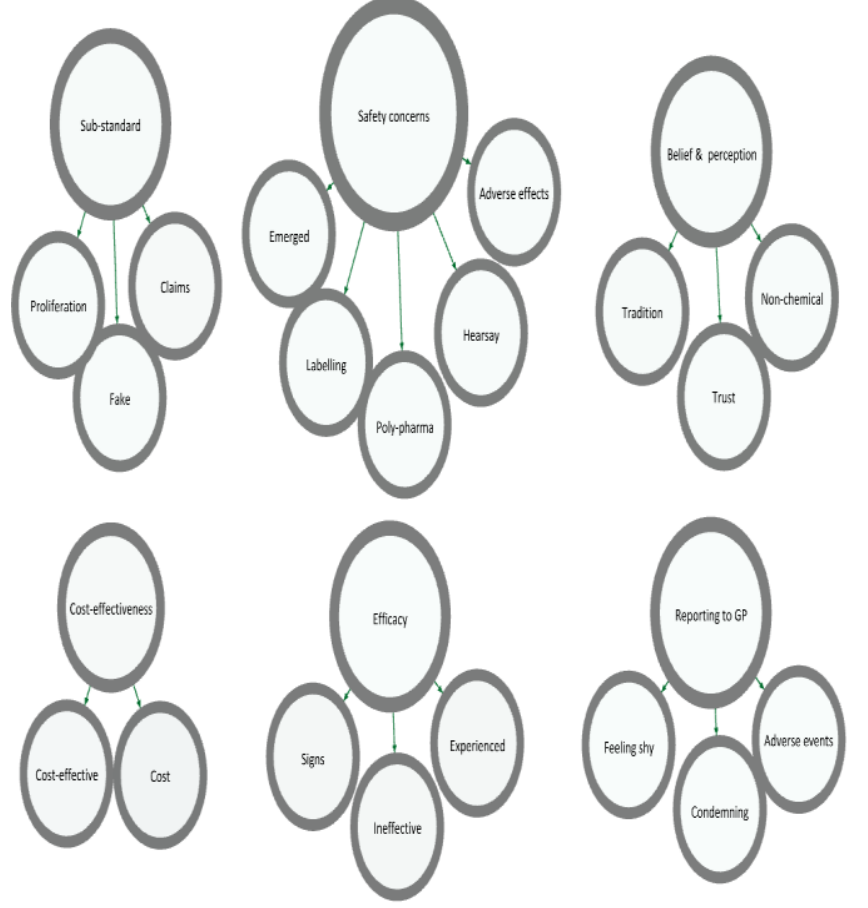

Figure 1: Thematic map. A plot showing the connection between the codes (sub-themes) and code categories (themes) generated with Gephi. The distance between the vertices and the sizes of the vertices are not to scale. The vertices larger in size represents themes and the vertices smaller in size represent codes or subthemes in this study. The lines are edge lines representing connection between either code-code, code-code category or code category-code category connections. Key: GP represents General practitioner.

\section{Reporting adverse events to GP-(Adverse events)}

Though many interviewees (10) felt shy to freely tell their GP that they were using HMs, about 50\% were rather willing to report the side effects freely to the GP. Informants $\mathrm{H} 2$ and $\mathrm{H} 7$ commented that, they will report the side effects to the physician and they will not keep it to themselves, so that, the GP could give them guidance to relief the side effects. "You need to (report the side effects), so that the doctor can guide you, and will help him also in the prognosis" (H22). "Yes, so that the information will enable the doctor to help me" (H33).

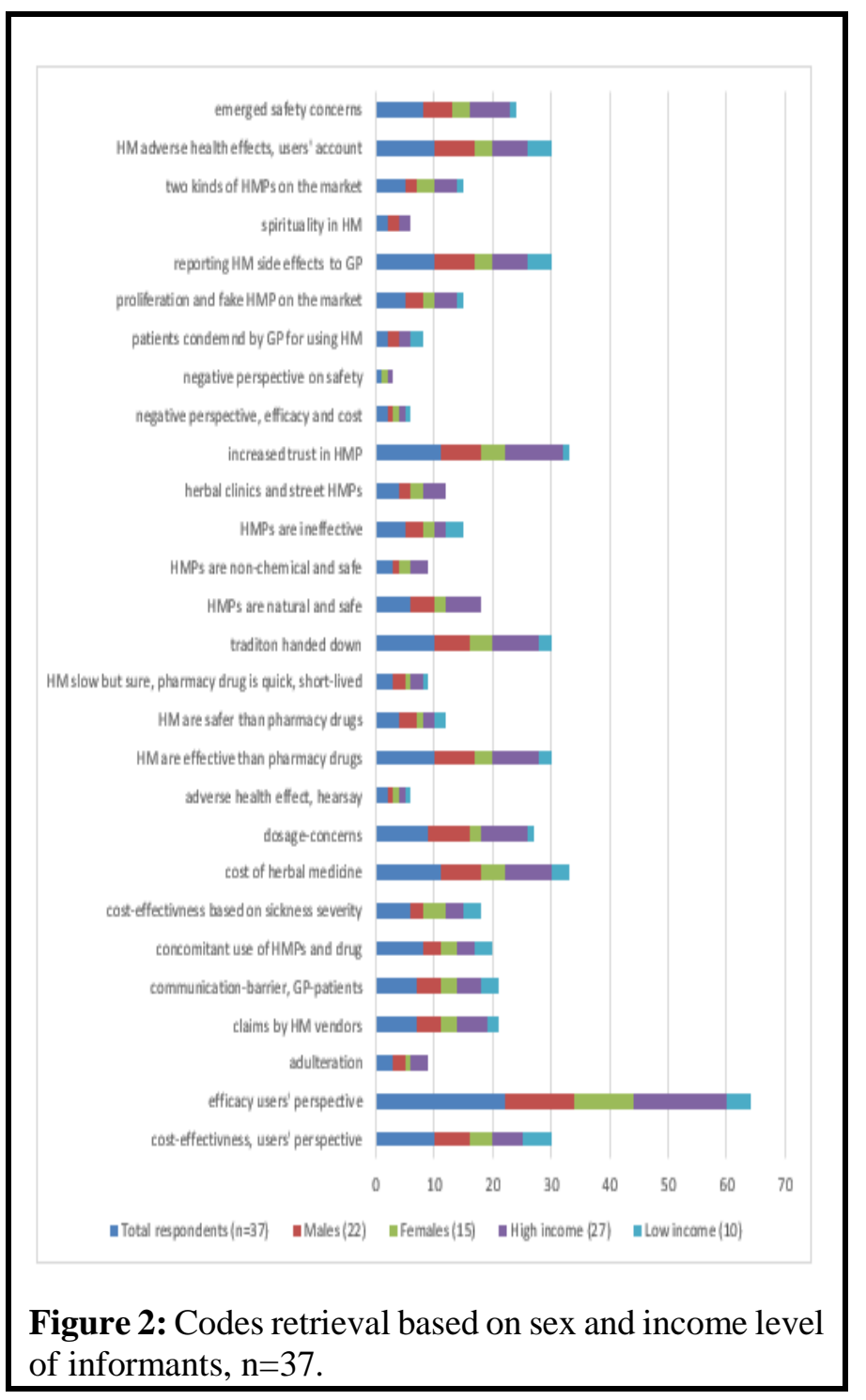

\section{Discussion}

In this study, we obtained some insight into the efficacy and safety of some commonly used herbal medicinal products (HMPs) in Kumasi, Ghana, from the perspectives of the users. This is relevant in informing or promoting rational use of HMP, improving public enlightenment and possibly informing coherent policy that will further promote the safe and rational use of HMs. In understanding the patient safety and use of 
herbal medicine from users' perspective, different themes and sub-themes emerged in this study, (Table 1, Figures 1 and 2).

It was observed that real-life expression of efficacy from the users' own feelings and physiological changes were relatively difficult, as well as affected by many factors. Many users vividly expressed what they meant by a medication being effective. To express HMPs efficacy, two interviewees used weird signs such as "sweating" or "increased sickness intensity". This expression just expanded the belief factor and tradition in herbal medicine (HM). In this study, some users believed good herbal preparations were more effective and safer than pharmaceutical drugs. This finding is consistent with a recent study in Ghana by Aziato and Antwi [21].

Evaluation or grading of efficacy from partial to very effective was difficult for informants except for one who had done laboratory tests after using HMP. According to her, her sugar level decreased but not as low as expected. This observation indicates the variation between assessing efficacy using patients' own senses and feelings than when using scientific methods. HMs' better effectiveness contradicts available scientific evidence [18]; less than $20 \%$ of the over 2000 herbal preparations and supplements were found by Bent to be effective scientifically. Recent research [6], identified that the efficacies of all the top5 antimalarial herbal preparations used in Central, Western and Greater Accra regions of Ghana were below WHO standard. Though the HMPs all reduced the level of parasitaemia to some extent, they were inadequate to be considered effective by WHO. This efficacy variation could partly be due to the holistic nature of the effect of HMPs compared to the targeted pharmaceutical drugs. Most scientific methods are designed to assess efficacy using parameters including specific cells, organs, tissues and pathogens. It is therefore recommended that further studies be carried out to assess the cause of the inconsistencies in the efficacies between the HMPs and the pharmaceutical drugs and to come out with a rigorous real-life assessment method of the efficacy of HMs to get the real-life picture of these medications.

Most participants in this study believed HMs were moderately expensive; some being more expensive than pharmaceutical medicines. A similar trend was identified in Australia by Mcintyre et al [22]. This finding contradicts WHO position on HMs, which considers the use of HMs to be an affordable means of treatment [23]. This expense could be due to the subsidization of essential medicines in Ghana by international donors and the free provision of pharmaceutical medicines under the National Health Insurance Scheme (NHIS). The scheme provides free pharmaceutical medicines to patients under its coverage. HMPs did not benefit from any of these policies and might be considered expensive.

According to experiences shared by users, HMs are seen to be a 'slow but sure' way of treatment. They believe HMs are slow but can cure diseases while pharmaceutical medications are quick but best used to manage not necessarily cure diseases. Mcintyre and colleagues identified that this 'slow but sure' belief deters some patients from using HMs [22]. Aziato and Antwi [21] on the other hand, found this belief to be a motivator for some Ghanaians to use HMs. In this study, however, users saw it as a motivator and this correlated well with Aziato and Antwi's findings. This belief could partly explain why some users use HMs after pharmaceutical drugs administration to get the quick effects from the pharmaceutical drug and to "cure the health problem' with the HMs. Herb-pharmaceutical combined drug use were very common among the interviewees. This study emphasizes that some sicknesses can only be managed or treated with HMs. The dissatisfaction of some Ghanaians with the outcome from pharmaceutical medicines were identified in this study as some of the important factors increasing people's faith in HMs. The finding correlates with that of Peltzer and colleagues [3] who found a similar trend of increased trust in HMs.

Adulteration is a common but serious problem in herbal medicine worldwide, whereby HMPs are found to contain synthetic prescription drugs as chemical adulterants intentionally introduced [24]. Fake herbal products are those that have either been wholly or partly substituted with less/ inactive substances, synthetic drugs or are mislabelled. The practice is done by producers with the idea of improving the efficacy, misinform the public and to sell sham or inactive herbal products to the public. The illegal production, sale and distribution of fake drugs represents over $50 \%$ of the pharmaceutical market in several African countries [25]. Fake HMPs on the market were a major concern to many in this study, which adds to the global health problem of fake medications, in both pharmaceutical and herbal medicines alike [24]. The awareness of fake preparations in the market led many users in this study, to be cautious of the efficacy of herbal medicine. Thus, HMPs are effective when one gets unadulterated versions from the market. 
Some users expressed dissatisfaction with the market flooded with herbal preparations. The surge in HMPs could be due to market availability and increased reliance on herbal medicine. It might also be due to the urge to come out with new herbal products to combat the ever-increasing diseases affecting the public. Some users made the assertion that HMs are non-chemical, naturally extracted and are safe. This is an erroneous belief and it contradicts available scientific knowledge $[14,15,26,27]$. Adverse health effects including chronic kidney disease, stomach swollen, constipation, nausea, headache and dizziness were reported among some informants in this study. The findings expand the evidence that HMs are generally safe, but they could cause adverse health problems [7].

It was observed in this study that HM users find it difficult to voluntarily tell the general practitioners (GP) of their usage of HMs during hospital visits. This finding is in conformity with previous studies [22]. Two reasons were identified in this study to be the possible causes of the communication barrier between the physicians and the HM users; the first is "shyness" on the part of the users and the second is the "feeling of being condemned by the physician". Shyness, because in the past Ghanaians associated HM with spirituality and a HM user was believed to be a believer of those traditions or spiritualism. Such beliefs and perceptions are changing in recent years giving rise to an increase in patronage among the old and the youth (mean age of users in this study was $47.8 \pm 14.2$ and participants ranged from 23 to 80 years). The feeling of being condemned by physicians might be due to users' previous experiences with physicians on this matter or probably just a wrong notion about the physicians. Physicians can initiate discussion on this topic during visitations and guide users on the safe use of their herbal preparations since HM has become a fabric in the health care system in Ghana and has come to stay.

\section{Conclusion}

This study has provided some insight into the real-life experiences of selected Ghanaians with some of the commonly used HMPs in Kumasi. "Shyness" and the "feeling of being condemned by the physician" were identified to be the possible causes of the communication barrier between the physicians and the HM users. Concomitant use of HMPs and orthodox drugs was common especially among patients with chronic diseases and for treatment of malaria. Observed adverse effects based on informants' experiences were generally non-severe and minimal. Only $5.4 \%$ of participants had experienced moderate to severe adverse effects. We recommend physicians to initiate discussion on this topic during consultations and guide users on safe use of these HMPs. In the face of perceived proliferation and adulteration of HMPs as reported by users in this study, regulatory authorities need to bring some sanity into the herbal industry in Ghana. While most informants claimed HMs were generally safe and effective, others had unpalatable experiences with serious adverse health effects. We recommend therefore that large-scale post-market surveillance is conducted throughout the entire country regularly for proper documentation of safety, efficacy and adverse events on all HMPs on the market. We also recommend that all HMPs should be subjected to scientific evaluation for efficacy and safety prior to registration or approval by the Food and Drugs Authority (FDA) while we also continue to advocate the cautious use of all HMPs on the market.

\section{Acknowledgements}

The authors gratefully acknowledge the enormous assistance provided by Mr. Isaac Asirifi and all members of staff of the Department of Clinical Biochemistry of the Komfo Anokye Teaching Hospital, Kumasi, Ghana.

\section{Conflicts of Interests}

None declared.

\section{Funding}

None declared.

\section{Ethical Clearance and Consent to Participate}

Ethical clearance for the study was issued by the University of Cape Coast Institutional Review Board (UCCIRB) (ethical approval number: UCCIRB/EXT/2017/07). Individual consent to participate was sought from all participants. Individuals who agreed to participate in the study gave their consent by signing the consent form.

\section{Authors Contributions}

FA-M, ME and DG were responsible for conception and design of study. Data acquisition was carried out by FAM, ITH and ASEE. Analysis and interpretation of data were carried out by FA-M and SAO. Manuscript was drafted by FA-M. Revision of the manuscript for important intellectual content and final approval of the version to be published was done by AH, JK, CTK and ME. 


\section{References}

1. Boateng MA, Danso-Appiah A, Turkson BK, et al. Integrating biomedical and herbal medicine in Ghana-experiences from the Kumasi South Hospital: A qualitative study. BMC Complement Altern Med 2016; 16.

2. World Health Organization, WHO traditional medicine strategy: 2014-2023.

3. Peltzer K, Khoza LB, Lekhuleni ME, et al. Concepts and treatment modalities for hypertension by traditional and faith healers in the Northern Province, South Africa. Health SA Gesondheid 2001; 6: 59-67.

4. Hughes GD, Aboyade OM, Clark BL, et al. The prevalence of traditional herbal medicine use among hypertensives living in South African communities. BMC Complement Altern Med 2013; 13: 38 .

5. Bent S, Padula A, Neuhaus J. Safety and efficacy of citrus aurantium for weight loss. Am J Cardiol 2004; 94: 1359-1361.

6. Wilmot D, Ameyaw EO, Amoako-Sakyi D, et al. In vivo efficacy of top five surveyed Ghanaian herbal anti-malarial products. Malar J 2017; 16: 103.

7. Ernst E. Harmless herbs? A review of the recent literature. Am J Med 1998; 104: 170-178.

8. Ekor M. The growing use of herbal medicines: Issues relating to adverse reactions and challenges in monitoring safety. Front Pharmacol 2014; 4.

9. Harkey MR, Henderson GL, Gershwin ME, et al. Variability in commercial ginseng products: An analysis of 25 preparations. Am J Clin Nutr 2001; 73: 1101-1106.

10. Nortier JL, Martinez MC, Schmeiser HH, et al. Urothelial carcinoma associated with the use of a Chinese herb (Aristolochia fangchi). N Engl J Med 2000; 342: 1686-1692.

11. Ko RJ. Adulterants in asian patent medicines. N Engl J Med 1998; 339: 847-847.

12. Boullata JI, Nace AM. Safety issues with herbal medicine. Pharmacotherapy 2000; 20: 257-269.

13. Skoulidis F, Alexander GJM, Davies SE. Ma huang associated acute liver failure requiring liver transplantation. Eur J Gastroenterol Hepatol 2005; 17: $581-584$.
14. Nie J, Wang F, Ji T, et al. Assessment of in vitro cardiotoxicity of extract fractions and diterpene alkaloids from aconitum leucostomum Worosch: A short communication. J Pharm Biomed Anal 2017; 137: 84-89.

15. Navarro VJ, Barnhart H, Bonkovsky HL, et al. Liver injury from herbals and dietary supplements in the U.S. Drug-Induced Liver Injury Network. Hepatol Baltim Md 2014; 60: 1399-1408.

16. Yang L-X, Liu C-Y, Zhang L-L, et al. Clinical characteristics of patients with drug-induced liver injury. Chin Med J (Engl) 2017; 130: 160-164.

17. Nkansah MA, Opoku F, Ackumey AA. Risk assessment of mineral and heavy metal content of selected tea products from the Ghanaian market. Environ Monit Assess 2016; 188: 332.

18. Bent S. Herbal medicine in the United States: Review of efficacy, safety, and regulation. J Gen Intern Med 2008; 23: 854-859.

19. Braun V, Clarke V. Using thematic analysis in psychology. Qual Res Psychol 2006; 3: 77-101.

20. Content analysis and thematic analysis: Implications for conducting a qualitative descriptive study-Vaismoradi, 2013. Nursing \&amp; Health Sciences - Wiley Online Library.

21. Aziato L, Antwi HO. Facilitators and barriers of herbal medicine use in Accra, Ghana: An inductive exploratory study. BMC Complement Altern Med 2016; 16: 142.

22. McIntyre E, Saliba AJ, Moran CC. Herbal medicine use in adults who experience anxiety: A qualitative exploration. Int $\mathbf{J}$ Qual Stud Health Well-Being 2015; 10: 29275.

23. Guidelines for the Appropriate use of Herbal Medicines n.d.

24. Roger B, Boateng K. Bad medicine in the market. World Hosp Health Serv Off J Int Hosp Fed 2007; 43: 17-21.

25. Ambroise-Thomas P. The tragedy caused by fake antimalarial drugs. Mediterr J Hematol Infect Dis 2012; 4.

26. Adusei-Mensah F, Essumang DK, Agjei RO, et al. Heavy metal content and health risk assessment of commonly patronized herbal medicinal preparations from the Kumasi metropolis of Ghana. J Environ Health Sci Eng 2019. 
Citation: Frank AM, Ari H, Jussi K, et al. Post-Market Safety and Efficacy Surveillance of Herbal Medicinal Products from Users' Perspective: A Qualitative Semi-Structured Interview Study in Kumasi, Ghana. Int J Pharm Pharmacol 2019; 3: 136. doi: 10.31531/2581-3080.1000136

27. Adusei-Mensah F, Henneh IT, Ekor M. Pesticide residue and health risk analysis of six commonly used herbal medicinal products in Kumasi, Ghana. Texila Int J Public Health 2018; 6: 186-195.
28. Yadav P, Stigall K, Johnson HE, et al. Functional foods: How functional are they? A case report of supplement-induced psychosis. Int $\mathrm{J}$ Psychiatry Med 2016; 51: 479-485.

This manuscript was peer-reviewed

Mode of Review: Single-blinded

Academic Editor: Dr. MA Jahangir

Copyright: (02019 Frank AM, et al. This article is distributed under the terms of the Creative Commons Attribution 4.0 International License (http://creativecommons.org/licenses/by/4.0/), which permits unrestricted use, distribution, and reproduction in any medium, provided you give appropriate credit to the original author(s) and the source, provide a link to the Creative Commons license, and indicate if changes were made. 